\title{
Localization of musicogenic epilepsy to Heschl's gyrus and superior temporal plane: case report
}

\author{
Yasunori Nagahama, MD, ${ }^{1}$ Christopher K. Kovach, PhD, ${ }^{1}$ Michael Ciliberto, MD, ${ }^{2}$ \\ Charuta Joshi, MBBS, ${ }^{2}$ Ariane E. Rhone, PhD, ${ }^{1}$ Adam Vesole, BBA, ${ }^{3}$ Phillip E. Gander, PhD, ${ }^{1}$ \\ Kirill V. Nourski, MD, PhD, ${ }^{1}$ Hiroyuki Oya, MD, ${ }^{1}$ Matthew A. Howard III, MD, ${ }^{1,4}$ Hiroto Kawasaki, MD, ${ }^{1}$ \\ and Brian J. Dlouhy, MD ${ }^{1,4}$
}

Departments of ${ }^{1}$ Neurosurgery and ${ }^{2}$ Pediatrics, and ${ }^{3}$ Carver College of Medicine, University of lowa Hospitals and Clinics; and ${ }^{4}$ Pappajohn Biomedical Institute, University of lowa Carver College of Medicine, lowa City, lowa

\begin{abstract}
Musicogenic epilepsy (ME) is an extremely rare form of the disorder that is provoked by listening to or playing music, and it has been localized to the temporal lobe. The number of reported cases of ME in which intracranial electroencephalography (iEEG) has been used for seizure focus localization is extremely small, especially with coverage of the superior temporal plane (STP) and specifically, Heschl's gyrus (HG). The authors describe the case of a 17-year-old boy with a history of medically intractable ME who underwent iEEG monitoring that involved significant frontotemporal coverage as well as coverage of the STP with an HG depth electrode anteriorly and a planum temporale depth electrode posteriorly. Five seizures occurred during the monitoring period, and a seizure onset zone was localized to HG and the STP. The patient subsequently underwent right temporal neocortical resection, involving the STP and including HG, with preservation of the mesial temporal structures. The patient remains seizure free 1 year postoperatively. To the authors' knowledge, this is the first reported case of ME in which the seizure focus has been localized to HG and the STP with iEEG monitoring. The authors review the literature on $\mathrm{EEEG}$ findings in ME, explain their approach to HG depth electrode placement, and discuss the utility of STP depth electrodes in temporal lobe epilepsy.
\end{abstract}

https://thejns.org/doi/abs/10.3171/2017.3.JNS162559

KEY WORDS musicogenic epilepsy; Heschl's gyrus depth electrode; intracranial electroencephalography; superior temporal plane; medically refractory epilepsy

$\mathrm{M}$ USICOGENIC epilepsy (ME) represents a rare form of epilepsy provoked by listening to or playing music, with a prevalence of 1 per $10,000,000$ population. $1,5,7,14$ Various simple and complex auditory stimuli have been described as triggers for the seizures, and these auditory stimuli can be as specific as particular portions of particular songs by particular artists. ${ }^{1,7,14}$ The seizure onset zone of ME has been localized most frequently to the temporal lobe (with a slight bias toward the right hemisphere), predominantly by using surface electroencephalography (EEG). ${ }^{1,7,14}$

Intracranial EEG (iEEG) has the advantages of high spatial and temporal specificity in investigating seizure onset and propagation, which more common noninvasive studies, such as scalp EEG, lack. However, iEEG has been used in only a small number of reported ME cases. ${ }^{3,4,6,9-13}$ Previous iEEG reports have localized the seizure onset focus in ME either to the mesial temporal structures or to the advanced auditory areas in the planum temporale (PT) or lateral temporal cortex.

We describe a 17-year-old boy with a history of medically intractable ME involving the right temporal lobe who underwent iEEG monitoring. The seizure onset zone localized to Heschl's gyrus (HG) and the superior temporal plane (STP). The patient subsequently underwent seizure focus resection involving the STP, including $\mathrm{HG}$, but not

ABBREVIATIONS AED = antiepileptic drug; $A T L=$ anterior temporal lobectomy; EEG = electroencephalography; $H G$ = Heschl's gyrus; iEEG = intracranial EEG; ME = musicogenic epilepsy; $\mathrm{PT}=$ planum temporale; $\mathrm{STG}=$ superior temporal gyrus; $\mathrm{STP}=$ superior temporal plane.

SUBMITTED October 7, 2016. ACCEPTED March 17, 2017.

INCLUDE WHEN CITING Published online September 15, 2017; DOI: 10.3171/2017.3.JNS162559. 

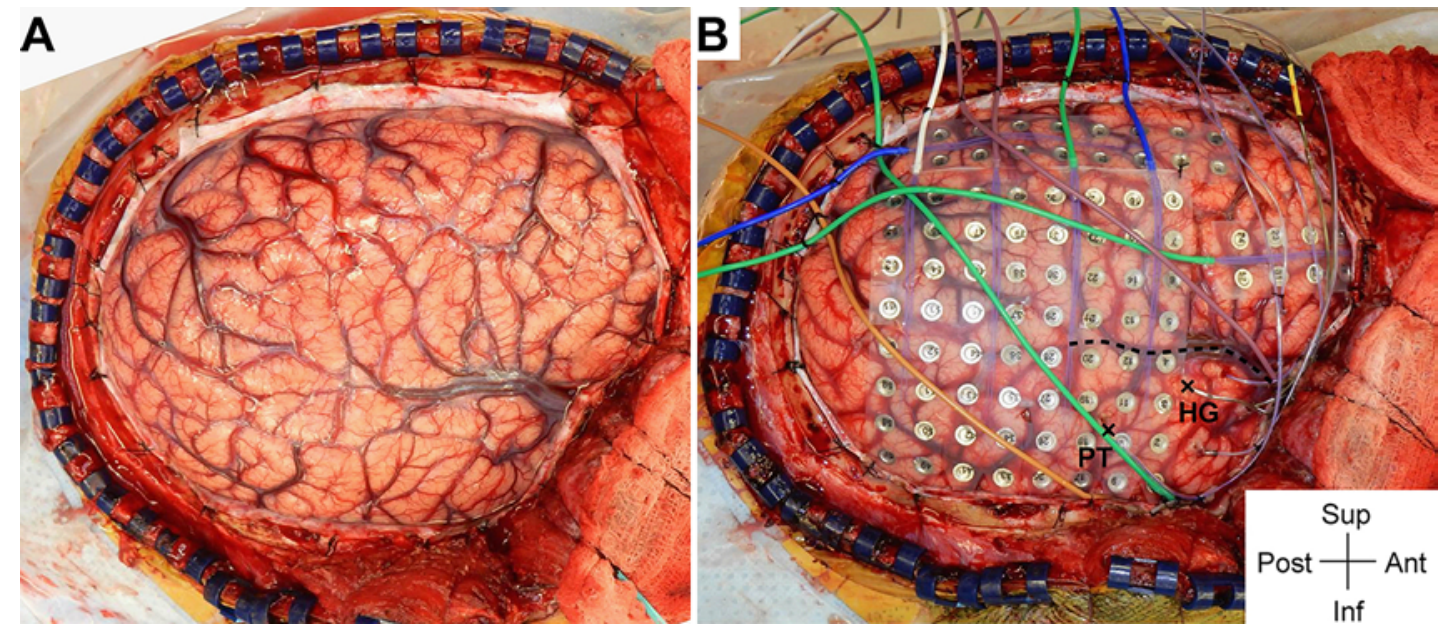

FIG. 1. Intraoperative photographs demonstrating exposure and intracranial electrode placement. A right frontotemporoparietal craniotomy (A) allowed proper exposure for placement of grid, strip, and depth electrodes (B), including the HG depth electrode. The sylvian fissure is marked with a dashed line. The HG depth electrode and the PT depth electrode are marked with X symbols anteriorly and posteriorly, respectively, at their entry points at the cortical surface. . Ant = anterior; inf = inferior; post = posterior; sup $=$ superior. Figure is available in color online only.

the mesial temporal structures. We review the literature on iEEG findings in ME, explain our approach to HG depth electrode placement, and discuss the utility of STP depth electrodes in temporal lobe epilepsy.

\section{Case Report}

Presurgical Presentation and Evaluation

A 17-year-old right-handed boy began having complex partial seizures at 11 years of age. Seizure semiology involved a sensation of hearing indescribably distorted and rhythmic sounds, which became more distorted with longer and more extensive seizures. During seizures, his speech would sometimes become altered, which he was not aware of, and he would sometimes lose awareness of himself. These seizures did not have an emotional component, nor were they triggered by any emotional content. Most were simple partial seizures or had dyscognitive features. Generalized tonic-clonic seizures were rare, with only 5 occurring over 6 years. The seizures were often triggered by various sounds, songs, or rhythms, although the patient was not able to identify any particular auditory stimuli that consistently evoked seizures. He played the viola, was in his school's orchestra, and often had seizures triggered during orchestra practice. His seizures were initially well controlled with antiepileptic drugs (AEDs), but the AEDs ultimately failed and he began to have frequent breakthrough seizures ranging from several daily to a frequency of 1-2 times a week. In total, 5 AEDs failed to control his seizures.

Video-EEG studies suggested right neocortical temporal lobe epilepsy, although many of his auras did not have correlating EEG findings. Results of a 3-T MRI study of the brain were normal. A PET scan with $\left[{ }^{18} \mathrm{~F}\right]$ fluorodeoxyglucose revealed no focal areas of hypometabolism. Magnetoencephalography showed significant spikes localized predominantly within the right superior temporal region extending from $\mathrm{HG}$ to the anterior-middle superior tem- poral gyrus (STG) and, to a lesser extent, in the adjacent inferior frontal gyrus and insula. The results of neuropsychological testing were not strongly lateralizing or localizing, and showed strong baseline cognitive functions.

\section{Electrode Implantation}

The patient underwent a right-sided frontotemporoparietal craniotomy for placement of intracranial electrodes (Fig. 1). Electrode coverage of the temporal and extratemporal areas was based on the following: 1) semiology of ME has shown a predilection for the temporal lobe, and video-EEG suggested a temporal lobe seizure focus; 2 ) there was a concern that the frontal lobe might be involved, based on prior EEG studies showing interictal discharges in the more superior and anterior areas (e.g., $\mathrm{C} 4$ and F4 areas); 3) many of the patient's auras did not have correlating EEG findings, and there were no focal lesions noted on high-resolution 3-T MRI (preoperatively and in retrospect) and no focal areas of hypometabolism on PET scans to guide electrode placement; 4) the results of the neuropsychological evaluation were not lateralizing or localizing to any specific areas; 5) coverage with the use of subdural grid and strip electrodes would potentially allow a more tailored resection depending on the pattern of ictal onset and propagation in the perisylvian areas after mapping of the motor area; and 6) the unique oblique orientation of $\mathrm{HG}$ and its proximity to the major perisylvian vasculature required exposure and direct visualization of the anterior perisylvian temporal area for placement of the HG depth electrode. Depth electrodes were placed using frameless stereotactic intraoperative image guidance, and targeted the STP, including HG and the PT, as well as the mesial temporal lobe (the amygdala and posterior hippocampus), the insula, and the inferior orbitofrontal area.

\section{Placement of HG Depth Electrode}

An HG depth electrode was placed using a frameless 

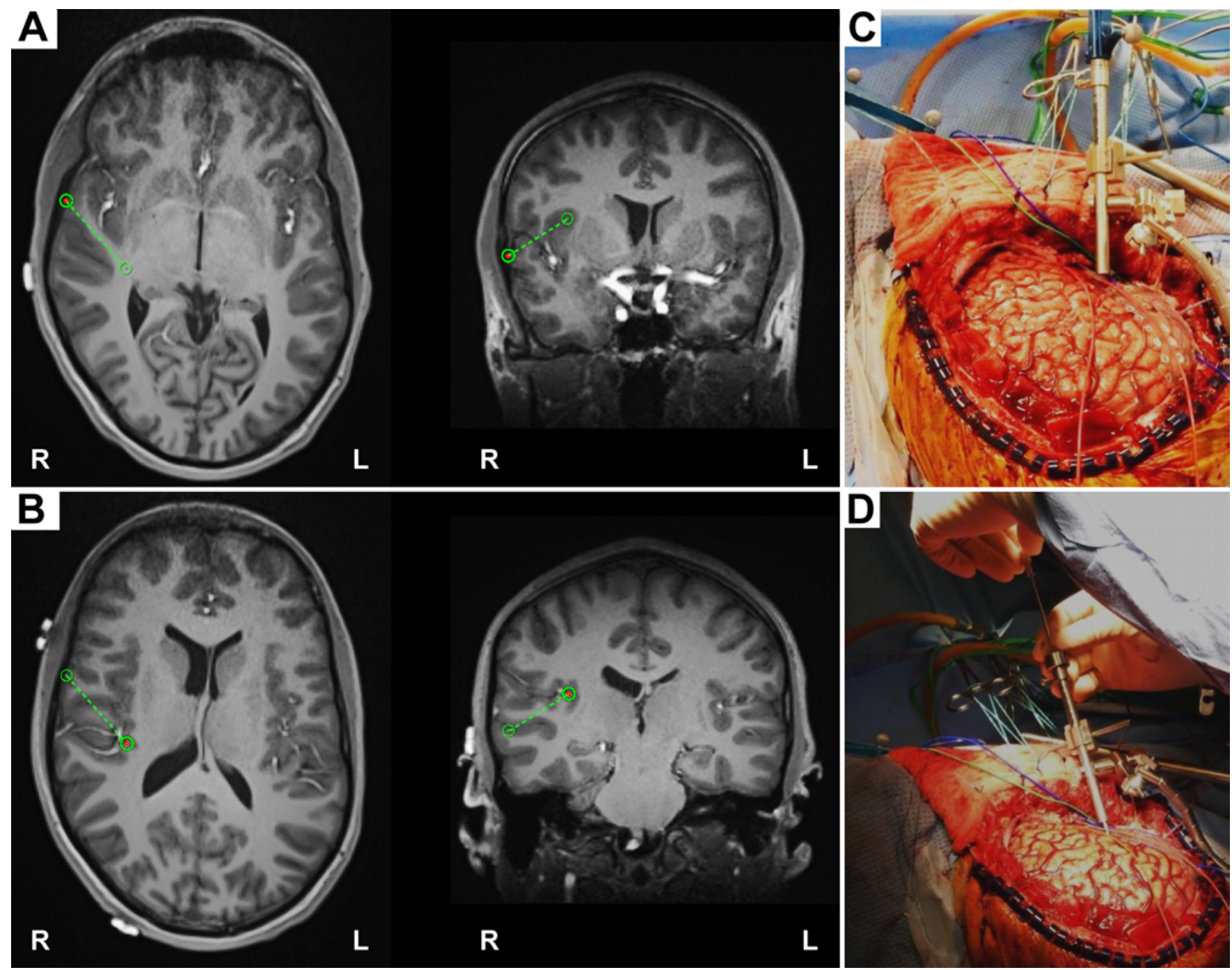

FIG. 2. Intraoperative placement of the HG depth electrode. A: The planning view on the frameless stereotactic system (Stealth Navigation, Medtronic) showing the entry point and the trajectory (green circles and dotted lines). B: The similar planning view showing the target and the trajectory. C and D: Intraoperative photographs showing placement of the HG depth electrode. A Stealth Navigus probe was used to select the appropriate trajectory of a guiding tube positioned over the entry point (C). An electrode-guiding cannula was advanced through the tube to the previously determined depth (D). An actual depth electrode was subsequently passed through the cannula, followed by removal of the guiding tube/cannula system. Note the unique anterolateralto-posteromedial trajectory within the STP for placement of the HG depth electrode. Figure is available in color online only.

stereotactic system (Stealth Navigation, Medtronic) as previously described, ${ }^{8}$ and will be briefly summarized here (Fig. 2). The HG, also known as the transverse temporal gyrus, is located in the STP and includes the primary auditory area in its posteromedial portion. Placement of an HG depth electrode presents a unique technical challenge because of the oblique orientation of $\mathrm{HG}$ relative to the sagittal and coronal plane and the proximity of $\mathrm{HG}$ to the branches of the middle cerebral artery within the sylvian fissure. Use of an HG depth electrode is considered in cases of temporal lobe epilepsy with possible lateral/ dorsal neocortical involvement and/or auditory symptoms. The STP coverage could be augmented with a PT depth electrode for more thorough coverage in complex cases. For HG depth electrode placement, an entry point and an end target point are chosen at the anterior lateral margin and the posterior medial margin, respectively, of the HG within the STP, with the distance typically measuring between 40 and $50 \mathrm{~mm}$. Given the oblique orientation of the $\mathrm{HG}$, exposure of the anterior perisylvian temporal area is needed for placement of the HG depth electrode.

\section{Results of iEEG Monitoring}

Over a 3-week monitoring period (extended due to lack of the habitual clinical seizures during the first 2 weeks, despite quick tapering of the AED within the 1st week of the monitoring), the patient had a total of 5 seizures, including one that was elicited while listening to a recording of Beethoven, with typical seizure semiology of sound distortion. During these episodes, significant spikes were consistently noted in the STP along HG and PT at seizure onset (Fig. 3). The amplitudes of the spikes were consis- 


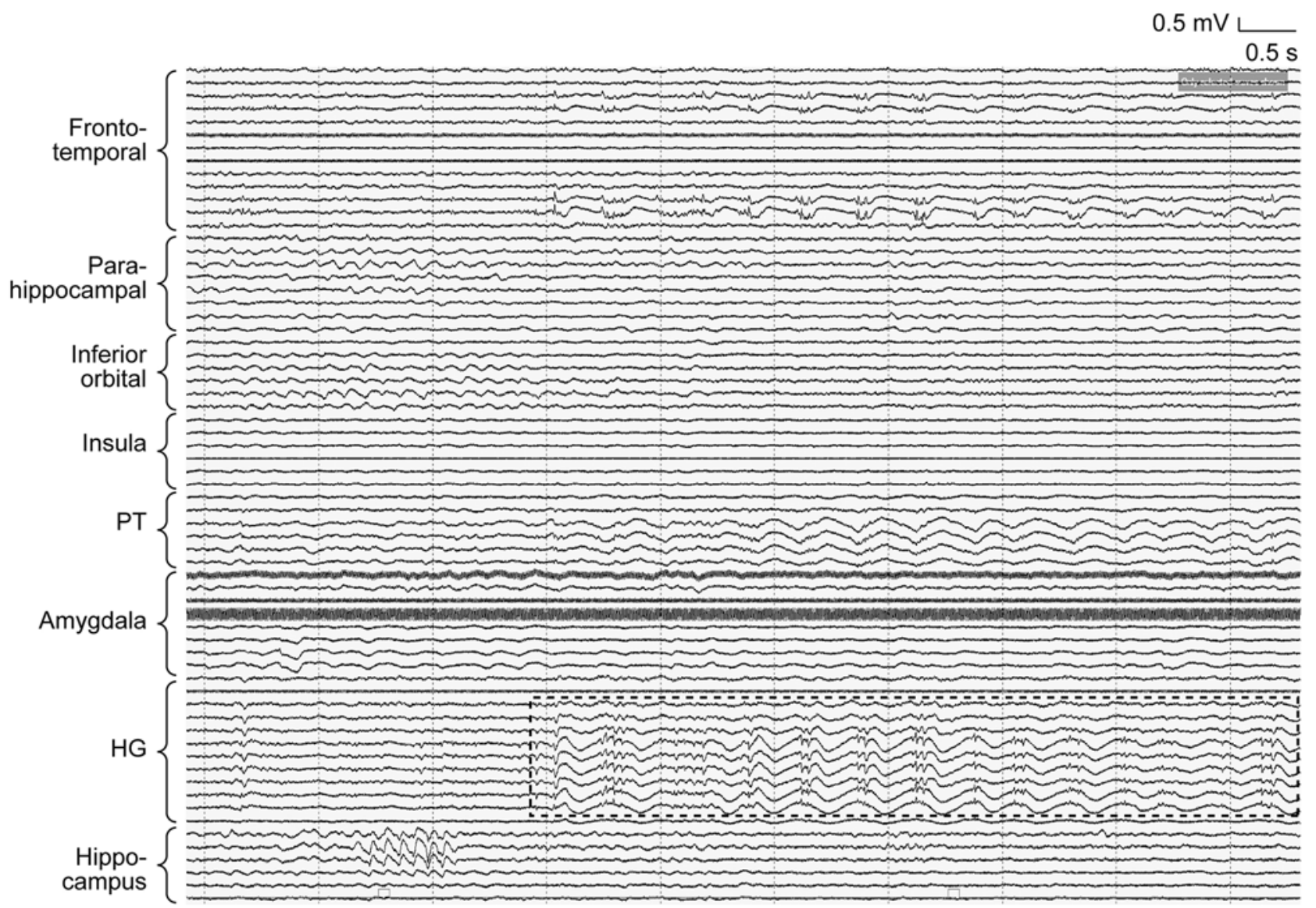

FIG. 3. Invasive EEG results showing significant spikes along the HG depth electrode at the onset of a typical seizure. The most significant (largest in amplitude) spikes were consistently noted along the HG depth electrode at the seizure onset (marked with a dashed rectangular area), followed by spikes of smaller amplitudes along the PT depth electrode. Spikes noted with the frontotemporal grid electrode over the lateral temporal cortex and centered around the entry points of the HG and PT depth electrodes were delayed in some cases by 1-2 msec, and were smaller in amplitude at seizure onset. No significant spikes were noted in the mesial temporal lobe with amygdala or posterior hippocampus depth electrodes or with a parahippocampal strip electrode. Neither an inferior orbital depth electrode nor an insula depth electrode was involved.

tently largest in HG. Onset of the spikes in HG consistently preceded that in PT. Spikes were also noted over the lateral temporal cortex centered on the area between the entry points for the HG and PT depth electrodes over the STG, but these spikes were delayed in some cases by 1-2 msec, and were smaller in amplitude at seizure onset. Rapid ictal propagation was noted from these areas to the more anterior and ventral temporal areas, including the temporal pole. Interictal discharges were observed to broadly involve the anterior temporal lobe. These results led us to strongly suspect that a seizure onset zone involved HG and the STP, with subsequent ictal propagation to extensively involve the anterior temporal cortex (Fig. 4). The mesial temporal structures, covered with 2 depth electrodes targeting the amygdala and posterior hippocampus, were never involved during seizure onset or propagation.

\section{Electrode Removal and Seizure Focus Resection}

After the epilepsy monitoring period the patient was taken back to the operating room for removal of the electrodes, followed by functional mapping of the primary motor cortex with somatosensory evoked potentials and motor evoked potentials (in case of future seizure recurrence and potential need for a further surgical intervention involving the frontal opercular area). The subsequent seizure focus resection involved the STP (including both HG and PT), using depth electrode contacts to guide the medial extent of resection as well as the lateral temporal cortex, temporal pole, and temporal base lateral to the collateral sulcus, while preserving the primary motor cortex, the frontal operculum, and the mesial temporal structures (Figs. 5 and 6). No additional areas were resected. The extensive resection involving the anterior lateral and ventral temporal cortex, including the temporal pole, was deemed necessary and indicated, given the interictal discharges broadly involving these areas and the rapid ictal propagation from HG and the STP broadly to the anterior temporal lobe.

\section{Postoperative Outcome}

The patient recovered well postoperatively, without observable neurological deficits. Resected temporal lobe pathology was consistent with focal cortical dysplasia Type I. The patient remains seizure free 1 year postoperatively, 

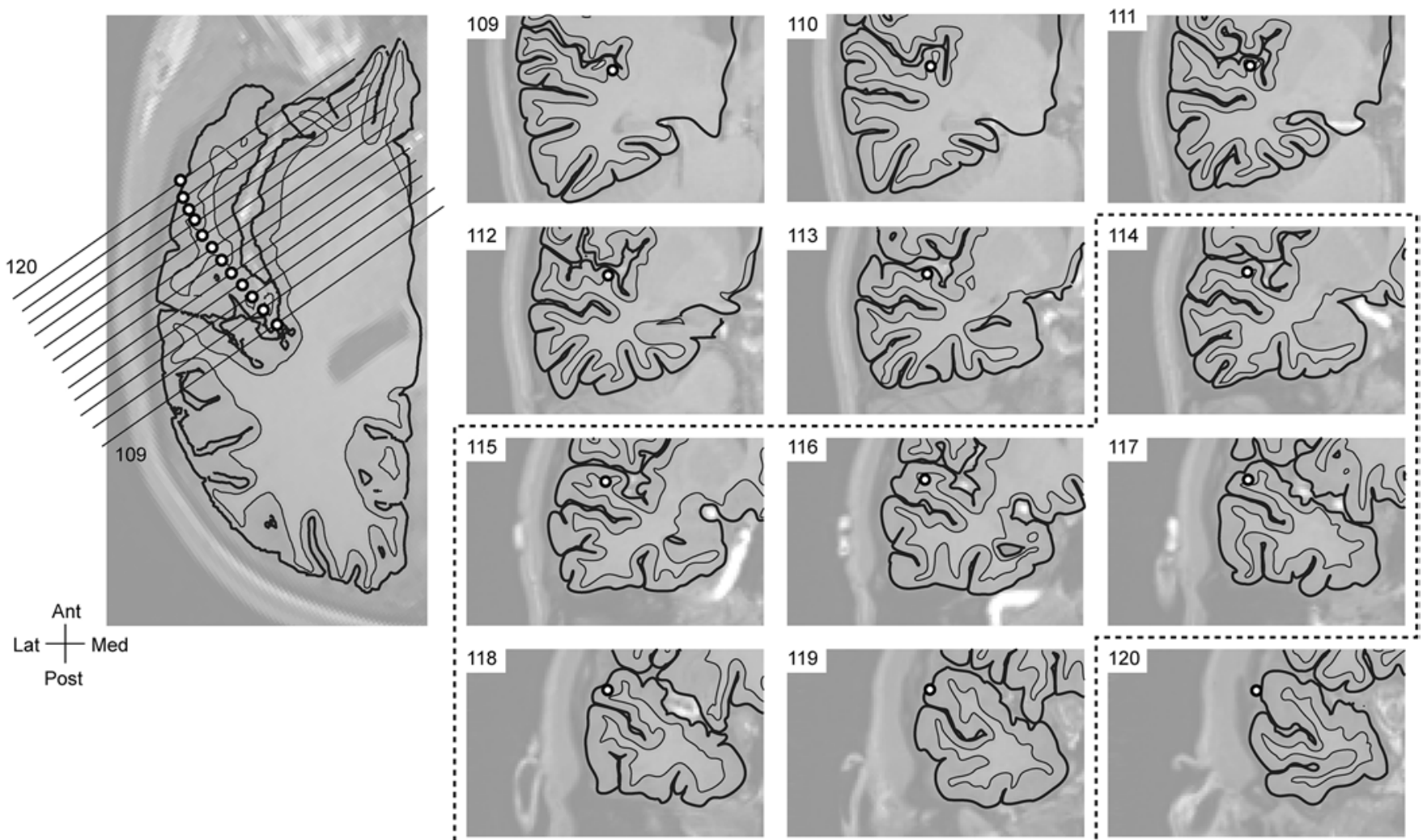

FIG. 4. The location of each contact of the HG depth electrode. The location of each contact (numbered from 109 posteromedially to 120 anterolaterally) is shown on the axial image on the left and on the coronal images on the right. The electrode contacts that showed significant spikes during the seizure onsets were marked with a dashed line (from contact 114 medially through contact 119 laterally).

and continues to be treated with the same preoperative AEDs. Postoperative neuropsychological testing demonstrated stable, strong cognitive functions, unchanged from the preoperative testing results, without evidence of new focal cognitive dysfunctions. The patient is back in school and enjoying playing the viola in his school's orchestra, without auditory deficits. He has not noted any subjective changes in his musical interest or appreciation and in his
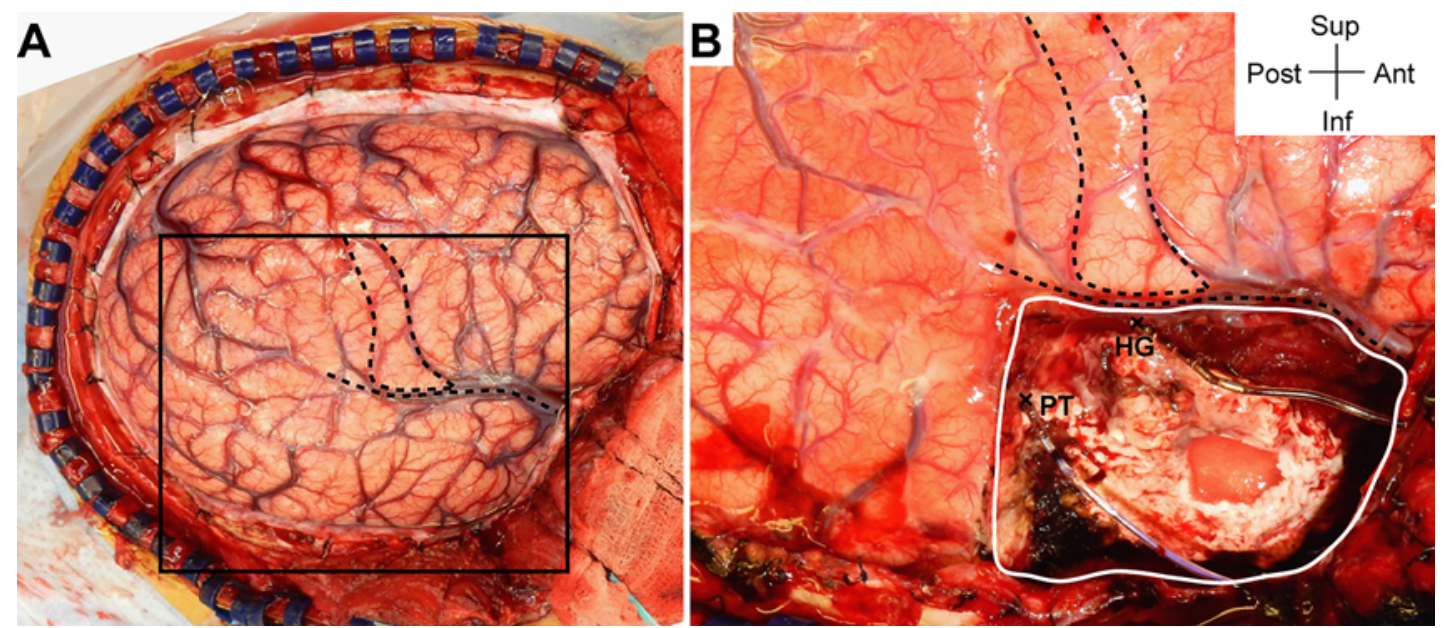

FIG. 5. Intraoperative photographs demonstrating resection of seizure focus. A: Right frontotemporoparietal craniotomy (same image as Fig. 1A). The box corresponds to the approximate area shown in panel B. Gross anatomical landmarks (primary motor area and sylvian fissure) are indicated by dashed lines. B: An expanded view showing the extent of resection visible in this view (solid white line) along with the HG depth electrode anteriorly and the PT depth electrode posteriorly that were kept in place during resection (entry points are marked by $X$ symbols). Figure is available in color online only. 


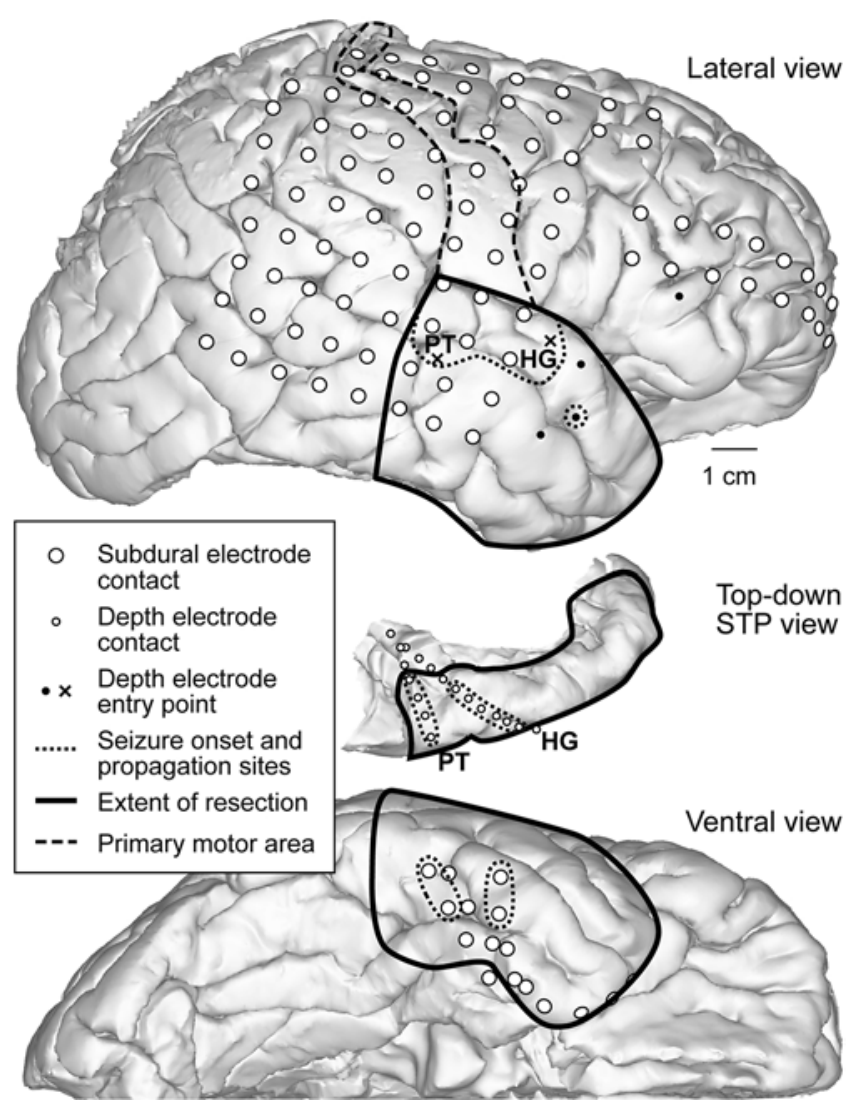

FIG. 6. Reconstructed brain images with electrode contacts, showing the locations of the most significantly involved contacts during seizure onset and propagation, the extent of resection, and the HG and PT depth electrodes relative to the STP. The surface views were created by the FreeSurfer software based on preoperative and postoperative imaging studies. The lateral (upper panel) and ventral (lower panel) views show electrode contacts (large white circles), areas most significantly involved in seizure onset and propagation (dotted lines), the mapped primary motor area (dashed line), the extent of resection (solid line), and the entry points for the $\mathrm{HG}$ and PT depth electrodes ( $X$ symbols and small black circles). The top-down view of the right STP (center panel) shows the locations of the HG and PT depth electrode contacts (small white circles).

ability to play the viola. The standard audiology evaluation revealed no auditory deficits bilaterally in hearing or discrimination across the normal hearing frequency range. Postoperative performance on speech perception tasks assessing /p/-/b/ and /s/-/sh/ discrimination (requiring auditory temporal and spectral acuity, respectively) was normal. The patient himself stated that "it was the greatest feeling ever to be able to simply listen to music and not have even the slightest feeling of a seizure."

\section{Review of the Literature}

We performed a literature review using the PubMed database, with search terms "musicogenic epilepsy" and "intracranial EEG," and identified 58 potentially relevant papers. The titles (as well as the abstracts when available) of all the identified papers were reviewed. The references in some of the more recent review papers were also reviewed. We ultimately identified 8 case reports that togeth- er described 10 cases of $\mathrm{ME}$ in which iEEG was used for seizure focus localization (Table 1). , $^{3,4,6,9-13}$

Overall, iEEG studies have been reported in 10 patients (4 male and 6 female patients), with the first case reported in 1999., 3,6,9-12 Eight of these patients had a seizure focus localized unilaterally to the temporal lobe (right temporal in 6 cases and left temporal in 2 cases). During seizure onset, the lateral temporal cortex was involved in 3 cases, the mesial temporal structures in 3 cases, the PT in 1 case, and both the lateral cortical and mesial structures in 1 case. All of these 8 patients subsequently underwent resective surgeries following the invasive monitoring. In particular, 4 patients underwent right anterior temporal lobectomy (ATL) that included the mesial temporal structures, 1 patient underwent right ATL that spared the hippocampus, 1 patient underwent right superior temporal lesionectomy, 1 patient underwent left superior temporal topectomy, and 1 patient underwent tailored resection of the left STG including the STP. Six of these patients achieved Engel Class I outcome, while the others reached Engel Class II outcome. The 2 patients who did not undergo resective surgeries were found to have bilateral mesial temporal onsets; one of these patients had a vagal nerve stimulator placed and achieved an Engel Class II outcome.

Among the 10 previously reported cases, depth electrodes were placed specifically in HG in 3 cases. ${ }^{6,10,13}$ One of these patients had a PT depth electrode as well as an HG depth electrode, and had a seizure focus localized specifically to PT, with subsequent seizure propagation to HG. ${ }^{13}$ The other 2 patients with HG coverage (1 of whom had 3 depth electrodes reportedly covering $\mathrm{HG}$ ) were noted to have right mesial temporal lobe seizure onsets, with subsequent propagation to HG. , $^{6,10}$

\section{Discussion}

Musicogenic epilepsy, a term first coined by Critchley, represents a rare form of epilepsy elicited by listening to or playing music, with a prevalence of 1 per 10,000,000 population. ${ }^{1,2,5,7,14}$ The role and characteristics of auditory stimuli as triggers for seizures in individual patients are variable. ${ }^{1,714}$ Seizures in some patients may be provoked exclusively by listening to music, whereas seizures in other individuals may be provoked by music and other nonauditory stimuli. Auditory triggers can be as specific as particular songs or particular portions of certain songs by particular artists, or they can be less specific and be described by types or categories of music, familiarity, and/or types of instruments. Some cases of ME have been characterized by specific emotional content of the triggering music. There may be a variable latency of up to several minutes between the auditory trigger and the seizure onset.

In the patient described in this report, seizures were elicited by listening to music, most notably during his orchestra practice. However, he was not able to identify specific music that would consistently elicit a seizure, and denied any specific emotional factors during the typical seizures. Furthermore, his seizures were not exclusively elicited by music. This is not unusual for cases of ME. ${ }^{14}$

Others have suggested an association between ME and the musical expertise of the patient. ${ }^{14}$ Interestingly, the pa- 
TABLE 1. Summary of 10 published cases with iEEG findings in patients with ME

\begin{tabular}{|c|c|c|c|c|c|c|}
\hline $\begin{array}{l}\text { Authors } \\
\& \text { Year }\end{array}$ & $\begin{array}{c}\text { Age } \\
\text { (yrs), } \\
\text { Sex }\end{array}$ & Triggers & HG Depth Electrode & Ictal Onset Zone & $\begin{array}{l}\text { Resection } \\
\text { Extent }\end{array}$ & $\begin{array}{c}\text { Outcome } \\
\text { (Engel } \\
\text { class) }\end{array}$ \\
\hline $\begin{array}{l}\text { Trevathan et al., } \\
1999\end{array}$ & $43, M$ & Specific theme songs & No & Rt STG & $\begin{array}{l}\text { Rt temporal lesion- } \\
\text { ectomy }\end{array}$ & I \\
\hline \multirow[t]{3}{*}{ Tayah et al., 2006} & $20, F$ & Popular music rhythms & No & Rt lateral neocortical temporal & $\begin{array}{l}\text { Rt ATL, spared } \\
\text { hippocampus }\end{array}$ & 1 \\
\hline & $24, F$ & Listen/play emotional songs & Yes (3 depth electrodes) & Rt mesial temporal & Rt ATL & I \\
\hline & $19, \mathrm{~F}$ & $\begin{array}{l}\text { Emotional church hymns \& } \\
\text { store music }\end{array}$ & No & $\begin{array}{l}\text { Bilat mesial temporal, inde- } \\
\text { pendently }\end{array}$ & No resection & NA \\
\hline Mehta et al., 2009 & $24, F$ & Rhythmic music & Yes & Rt mesial temporal & Rt ATL & I \\
\hline $\begin{array}{l}\text { Duanyu et al., } \\
2010\end{array}$ & $16, M$ & $\begin{array}{l}\text { Popular rhythms, singing } \\
\text { more effective }\end{array}$ & No & Lt STG (middle portion) & $\begin{array}{l}\text { Lt superior tempo- } \\
\text { ral topectomy }\end{array}$ & II \\
\hline Wang et al., 2012 & $42, \mathrm{M}$ & Specific songs & $\begin{array}{l}\text { Yes (w/ a PT depth } \\
\quad \text { electrode) }\end{array}$ & Lt PT & $\begin{array}{l}\text { Lt tailored STG } \\
\text { resection }\end{array}$ & I \\
\hline Tezer et al., 2014 & $33, F$ & $\begin{array}{l}\text { Affective music in native } \\
\text { language }\end{array}$ & No & $\begin{array}{l}\text { Bilat hippocampus (rt, musi- } \\
\text { cogenic; It, spontaneous) }\end{array}$ & $\begin{array}{l}\text { No resection (VNS } \\
\text { was placed) }\end{array}$ & $\|$ \\
\hline Klamer et al., 2015 & $22, \mathrm{M}$ & Rap music & No & Rt mesial temporal & Rt ATL & $\|$ \\
\hline Stern, 2015 & $46, \mathrm{~F}$ & Various musical genres & No & Rt mesial \& superior temporal & Rt ATL & I \\
\hline
\end{tabular}

NA = not applicable; VNS = vagal nerve stimulator.

tient with ME described here was an expert viola player, performing in a competitive school orchestra. Musicogenic epilepsy has been frequently localized to the temporal lobe, with a slight right hemispheric preponderance. . $^{1,7,14}$ Similarly, in the case presented here, seizures were localized to the right HG and STP.

There have only been a few reported cases in which iEEG has been used for seizure focus localization in ME (Table 1), ${ }^{3,4,6,9-13}$ In the previously reported 10 iEEG-monitored cases, a seizure focus was unilaterally or bilaterally localized either to the mesial temporal structures or to the advanced auditory areas in the PT or lateral temporal cortex. Depth electrodes were placed in the STP to cover HG in only 3 cases, although the descriptions of the specific electrode position were limited. ${ }^{6,10,13}$

The case presented here is the first reported one of ME in which seizure onset has been localized with iEEG to HG and the STP. This finding suggests that not only the mesial temporal structures, the advanced auditory areas in the PT, and the lateral temporal cortex, but also the primary auditory cortex in HG may be involved in seizure onset in some cases of ME. This is not surprising given the important role HG plays as the primary auditory area.

The variability across patients in the type of auditory trigger and the need for emotional musical content probably reflects involvement of the different auditory (STP and superior lateral temporal cortex) and emotional (mesial temporal lobe) neural networks during seizure onset and propagation. The involvement of mesial temporal lobe structures has been implicated, given the importance of auditory emotional factors in cases of ME. ${ }^{10,14}$ In a study by Tayah et al., in 3 cases of ME seizure, foci were localized with iEEG to different areas within the temporal lobe (i.e., the mesial vs lateral neocortical areas). ${ }^{10}$ Two patients had auditory triggers that involved an emotional component and the seizure foci were localized to the me- sial temporal lobe. In the other patient, an auditory trigger consisted of rhythmic music, and the seizure focus localized to the lateral neocortical area.

In the patient described in this report, seizures were never emotional in nature, which is consistent with a seizure onset zone localized to HG and the STP without involvement of the mesial temporal structures. These findings enabled us to perform a right temporal neocortical resection, with preservation of the mesial temporal structures.

The difference across patients in the complexity and specificity of auditory triggers may reflect involvement of the different components of the auditory network (i.e., the primary auditory area in HG vs more advanced auditory areas in the PT and STG). Patients reported by Trevathan et al., Duanyu et al., and Tayah et al., who had specific and complex auditory triggers for seizures, had seizure foci localized to the right STG. ${ }^{3,10,12}$ In another patient who also had specific and complex triggers and underwent iEEG using both an HG depth electrode and a PT depth electrode, the seizure focus localized to the PT but not HG ${ }^{13}$ In contrast, the seizure focus was localized to HG but not PT within the STP in the case presented here. The patient in our case had seizures triggered during orchestra practice but not by specific music. Therefore, with a seizure focus involving HG, seizures may be more likely to be provoked by auditory stimuli of various kinds and features (both complex and simple) that have similarities at the more basic auditory level.

This case illustrates the utility of anatomically placed STP depth electrodes (HG and PT) in localizing seizure onset and propagation of seizures in temporal lobe epilepsy. Additionally, these electrodes are useful during seizure focus resection because the electrode contacts can be used to guide the medial and posterior extent of the resection, both bordering on eloquent brain areas. Although 
no noticeable auditory deficits were found in our patient after partial resection of the STP involving HG and PT, the electrodes allow more tailored resection of the STP to minimize risks of postoperative speech/language deficits.

\section{Conclusions}

This is the first reported case of ME in which a seizure focus was localized to HG and STP based on iEEG findings. This case demonstrates that a tailored resective surgery in ME can be beneficial and that it can be performed without significant auditory deficits when a seizure focus is appropriately localized. Furthermore, a depth electrode in $\mathrm{HG}$ is useful in localization of temporal lobe seizures involving the STP. We speculate that the difference across patients in the complexity and specificity of auditory triggers for seizures may reflect involvement of the different components within the auditory network (i.e., the primary auditory area in HG vs advanced areas in the PT and STG).

\section{Acknowledgments}

Dr. Howard has received support from the Department of Health and Human Services, National Institutes of Health, National Institute on Deafness and Other Communication Disorders (Grant No. 5R01DC004290-17; Project title: Human Auditory Cortex Physiology). Dr. Dlouhy has received support from the Department of Health and Human Services, National Institutes of Health, National Institute of Neurological Disorders and Stroke (Grant No. 5K12NS080223; Project title: Transitioning Early Career Neurosurgeons to Scientific Independence; Amygdala Influence on Brainstem Control of Breathing).

\section{References}

1. Avanzini G: Musicogenic seizures. Ann N Y Acad Sci 999:95-102, 2003

2. Critchley M: Musicogenic epilepsy. Brain 60:13-27, 1937

3. Duanyu N, Yongjie L, Guojun Z, Lixin C, Liang Q: Surgical treatment for musicogenic epilepsy. J Clin Neurosci 17:127129,2010

4. Klamer S, Rona S, Elshahabi A, Lerche H, Braun C, Honegger J, et al: Multimodal effective connectivity analysis reveals seizure focus and propagation in musicogenic epilepsy. Neuroimage 113:70-77, 2015

5. Maguire MJ: Music and epilepsy: a critical review. Epilepsia 53:947-961, 2012

6. Mehta AD, Ettinger AB, Perrine K, Dhawan V, Patil A, Jain SK, et al: Seizure propagation in a patient with musicogenic epilepsy. Epilepsy Behav 14:421-424, 2009

7. Pittau F, Tinuper P, Bisulli F, Naldi I, Cortelli P, Bisulli A, et al: Videopolygraphic and functional MRI study of musicogenic epilepsy. A case report and literature review. Epilepsy Behav 13:685-692, 2008

8. Reddy CG, Dahdaleh NS, Albert G, Chen F, Hansen D, Nourski K, et al: A method for placing Heschl gyrus depth electrodes. J Neurosurg 112:1301-1307, 2010

9. Stern J: Musicogenic epilepsy. Handb Clin Neurol 129:469477, 2015

10. Tayah TF, Abou-Khalil B, Gilliam FG, Knowlton RC, Wushensky CA, Gallagher MJ: Musicogenic seizures can arise from multiple temporal lobe foci: intracranial EEG analyses of three patients. Epilepsia 47:1402-1406, 2006

11. Tezer FI, Bilginer B, Oguz KK, Saygi S: Musicogenic and spontaneous seizures: EEG analyses with hippocampal depth electrodes. Epileptic Disord 16:500-505, 2014

12. Trevathan E, Gewirtz RJ, Cibula JE, Schmitt FA: Musicogenic seizures of right superior temporal gyrus origin precipitated by the theme song from 'The X-Files'. Epilepsia 40 (Suppl 2):23, 1999 (Abstract)

13. Wang ZI, Jin K, Kakisaka Y, Burgess RC, Gonzalez-Martinez JA, Wang S, et al: Interconnections in superior temporal cortex revealed by musicogenic seizure propagation. J Neurol 259:2251-2254, 2012

14. Wieser HG, Hungerbühler H, Siegel AM, Buck A: Musicogenic epilepsy: review of the literature and case report with ictal single photon emission computed tomography. Epilepsia 38:200-207, 1997

\section{Disclosures}

The authors report no conflict of interest concerning the materials or methods used in this study or the findings specified in this paper.

\section{Author Contributions}

Conception and design: Nagahama, Dlouhy. Acquisition of data: Nagahama, Kovach, Rhone, Vesole, Gander, Nourski, Oya, Kawasaki, Dlouhy. Analysis and interpretation of data: Nagahama, Kovach, Ciliberto, Joshi, Rhone, Gander, Nourski, Oya, Kawasaki, Dlouhy. Drafting the article: Nagahama, Vesole, Dlouhy. Critically revising the article: all authors. Reviewed submitted version of manuscript: all authors. Approved the final version of the manuscript on behalf of all authors: Nagahama. Administrative/technical/material support: Kovach, Nourski. Study supervision: Kawasaki, Dlouhy.

\section{Correspondence}

Yasunori Nagahama, Department of Neurosurgery, University of Iowa Hospitals and Clinics, 200 Hawkins Dr., Iowa City, IA 52242. email: yasunori-nagahama@uiowa.edu. 